\title{
TIME SIMULATION METHODS FOR TESTING PROTOCOL SOFTWARE EMBEDDED IN COMMUNICATING SYSTEMS
}

\author{
J. Latvakoski, H. Honka \\ VTT Electronics, Kaitoväylä 1, P.O.Box 1100, FIN-90571, Oulu, Finland. Tel. +358 8551 \\ 2111, Fax.+35885512320,E-mail: juhani.latvakoski@ele.vtt.fi, hannu.honka@ele.vtt.fi
}

\begin{abstract}
This paper deals with time simulation methods that are applicable for testing protocol software embedded in communicating systems. The novelty of the presented approach is that simulation time is used to control the level of nondeterminism caused by the environment of the software under test. The test system is controlled in such a manner that external non-determinism is allowed, reduced, or eliminated according to the instructions given by the tester. The described methods have been evaluated in and applied to testing protocols for several standards. A TETRA terminal test system has been outlined as an example, demonstrating how time simulation can be integrated with a TTCN tester and target implementation generated from the SDL model.
\end{abstract}

Keywords: Embedded software, protocol testing, time simulation, SDL, TTCN

\section{INTRODUCTION}

The protocol software testing of products consisting embedded communicating systems can be carried out in product environment, laboratory environment, and on workstations. The focus of this paper is on workstation testing, which is here referred to as simulation-based testing of protocol software. This kind of software validation involves simulating the environment of the component or subsystem being assessed. Thus, the target operating system is emulated, while the $\mathrm{I} / \mathrm{O}$ interfaces, missing subsystems, and the communication network are simulated. Through the employment of this method in protocol integration testing, we have found out that it is the repeatability of tests that causes the most significant problems. The repeatability issue can be divided into such problem areas as controllability of testing, observability of testing and test re- 
sult analysis. On closer examination, these subproblems reveal rather complex interdependencies in terms of time-dependent behaviour and non-determinism.

Controllability denotes the ease of producing a specific output from a specific input. Lack of controllability, on the other hand, is viewed as an inability to control the global state of a distributed system, caused by uncontrolled local clocks and by the use of message queues. Asynchronous local clocks of separate nodes make debugging difficult, the stopping of one node will not affect the other nodes. This is likely to cause serious problems to the debugging control. Observability implies the ease of determining, whether specified inputs are affecting the outputs. Adequate observability is essential for monitoring the dynamic behaviour of the entire test system (outputs and states) and for analysing the test results. Some of the changes in time-dependent behaviour may be ascribed to the instrumentation (probe effect) used for observing the system behaviour during testing. Furthermore, there may not be enough time to produce traces on the lower layers, which limits the possibilities of testing. The analysis of test results can be based on the test case verdicts (ISO/IEC 9646 1991) and/or trace analysis (Sarikaya 1988, Bochmann \& Bellal 1990, Wvong 1991, Bochmann et al. 1991). When applying test case verdicts, nondeterminism may lead to an explosion of alternatives in TTCN behaviour trees. Thus, the order of observed events and their timing information may vary in an unpredictable way, which, however, may still be acceptable.

In this paper, we use the term internal non-determinism referring to the nondeterministic implementation of the software under test (Weiss 1988, Schütz 1990, Carver \& Tai 1991, Tai et al. 1991). External non-determinism, in turn, is triggered by the concurrent (and reactive) environment of the software (Kim et al. 1995). The internal behaviour of the software being tested can be serialised, so as to work out the deterministic variant of implementation (Tai 1985, Weiss 1988, Auer \& Korhonen 1995). Another approach involves using deterministic scheduling and synchronisation in the operating system implementation (Schütz 1990). Both of these approaches may require changes in the implementation in order to initially remove non-determinism. In this case, however, a set of serialisation variants of the original implementation is tested, instead of testing the original concurrent program.

The simulation time can be handled using local clocks (de la Puente et al. 1993, de la Puente et al. 1994), and relying on the Lamport (1978) synchronising algorithm, for example. It is also possible to manage simulation time based on the local clocks using conservative (Candy \& Misra 1979) or optimistic (Jefferson 1985) approaches (Fujimoto 1995). A single global simulation clock can also be applied (Billoir 1996). In addition, the advance of simulation time can be continuous or discrete (Taha 1988, Seila 1995). The instrumentation can be kept active in a real product so that its behaviour remains consistent during testing (Thome 1993, Liband 1995, Dotseth \& Kuzara 1993, 
Billoir 1996). Additional hardware and tools can be used for implementing non-intrusive monitoring. According to another, different approach, null virtual simulation time could be used in the execution of the instrumentation code parts.

We are proposing that the test system is controlled in such a manner that external non-determinism is allowed, reduced or eliminated according to the instructions given by the tester. The novelty of this approach is that the simulation time is used for controlling the level of non-determinism caused by the environment of the software under test.

\section{SIMULATION CONCEPT}

The software being tested consists of application software and system software, i.e. the operating system. In our approach, the target operating system has been emulated on top of the host operating system (Figure 1).

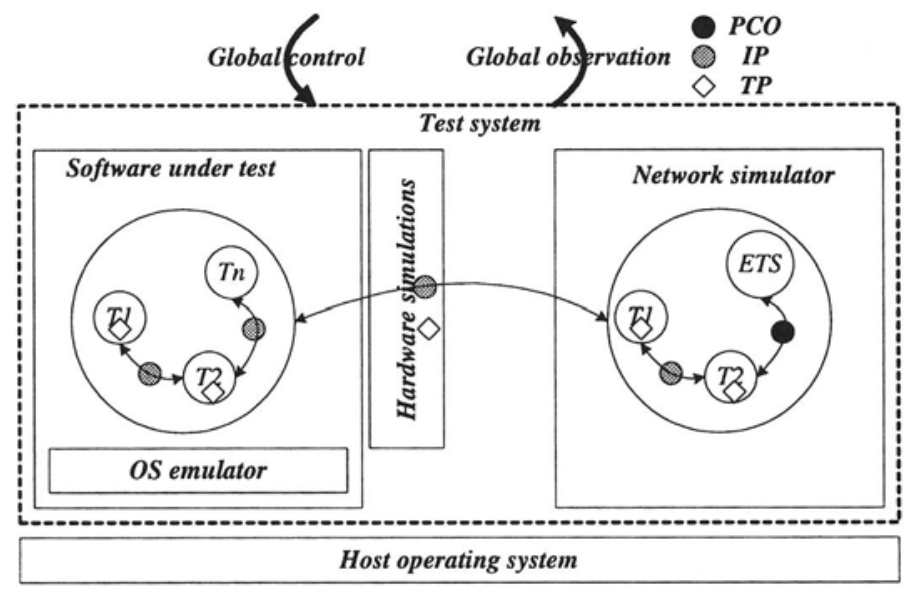

Figure 1 Test system.

Hardware simulations are required for simulating $\mathrm{V} / \mathrm{O}$, interrupts, and the behaviour of external hardware, such as processors and ASICs. The purpose of the network simulator is to implement a service provider for test cases. For example, when integrating the Ln, Ln-1, Ln- $2, \mathrm{Ln}-3, \ldots$, layers of a protocol stack, Ln-1, Ln-2, Ln-3, . . , the layers of the peer protocol need to be simulated. Test cases are designed to fulfil a specific test requirement (test purpose), and their implementation is shown as an executable test suite (ETS) in Figure 1.

The global control and observation mechanism applies the PCO concept of ISO. In addition, the interaction point (IP) has been designed to serve as a message passing interface between any modules or any real-time tasks of the test system, or any $\mathrm{I} / \mathrm{O}$ points between the software under test and its environment. At the test point (TP), some data items are changed and/or traced 
for testing purposes. The data may include an internal signal of a module or an $\mathrm{V} / \mathrm{O}$ signal between the software under test and its environment. A signal may consist of a block of data which does not include any message identifier, or alternatively, of a single piece of data or, finally, of no data at all. A message is a block of data characterised by a message identifier.

\section{TIME SIMULATION}

In this context, simulation time is equivalent to the behavioural time simulated for testing purposes. Tick, a simulated timer interrupt, serves here as a base unit, each tick increasing the time value by one. In our concept, the simulation time can be selected to be local or global (Figure 2). Simulation time is of the local type, if each node of the distributed test system has its own independent time reference. Accordingly, simulation time is global, if all the nodes share a common time reference.
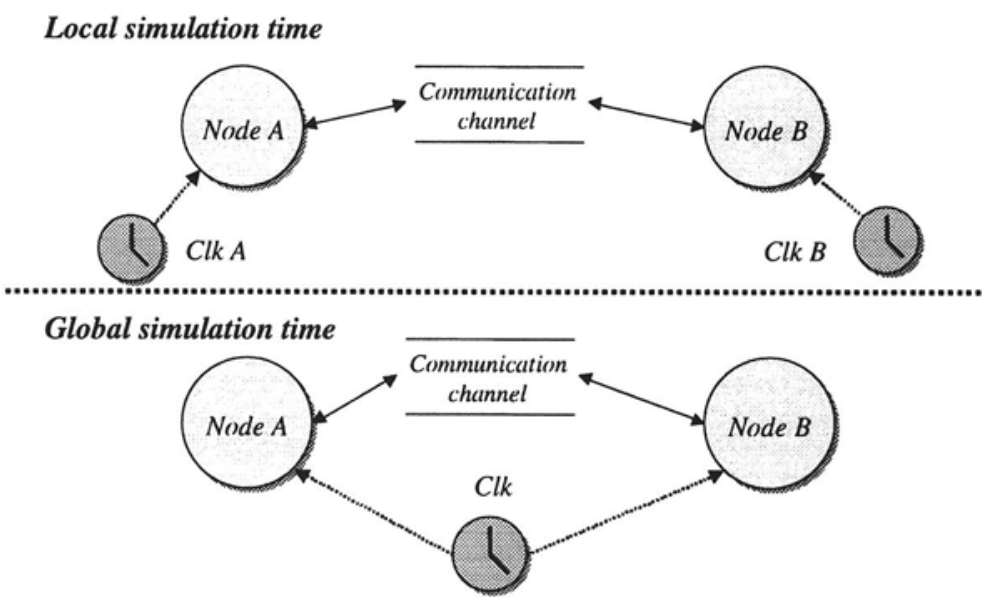

Figure 2 Local and global simulation time.

\subsection{BEHAVIOURAL TIME VS. REAL-TIME}

Behavioural time is based on timer services provided by the operating system. The smallest time unit of these timer services is usually a timer interrupt generated by the hardware or by a simulator. Real time, on the contrary, is based on the continuous advance of a real-time reference provided by an accurate hardware clock. The behaviour of the software under test is, naturally, dependent on real-time factors, including real-time delays of task execution times $(T e)$, real-time delays caused by the instrumentation, i.e., the probe effect $(T p)$, message passing delays in mailboxes $(T m b x)$, and message passing 
delays in the communication channels $(T c c)$ of the distributed simulation environment. Delays are also caused by the operating system due to the scheduling and synchronisation of real-time tasks (Tos), which, however, are not shown in Figure 3. In our approach, the real-time delays, such as $T e, T p, T m b x$, Tos and $T c c$, are included in the testing of performance constraints. The testing is carried out in the target hardware environment. However, these delays can be totally eliminated in the testing of the behavioural time-dependent features in a host by means of time simulation. When behavioural time is simulated, it can advance only when simulated timer interrupts occur. Task execution time $(T e)$, instrumentation $(T p)$, message passing in mailboxes $(T m b x)$, as well as the scheduling and synchronisation of real-time tasks (Tos) are assumed to be zero all the time. This is achieved by changing the real-time delay between two timer interrupts dynamically in such a way that there is just enough time for the software to carry out all the required operations before the next timer interrupt occurs. Similarly, the messages in the communication channels $(T c c)$ of the distributed simulation environment are transferred in null behavioural time. This is accomplished by keeping the order of timer interrupts and messages in the communication channels constant.

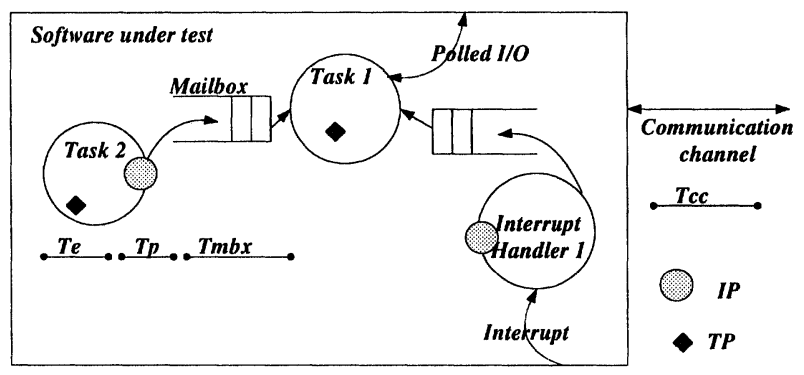

Figure 3 Time-dependent behaviour of a test system.

\subsection{CONTINUOUS VS. DISCRETE SIMULATION}

3.2.1 Continuous Simulation. The time reference (local or global) can be based on the clock of the host. In this case, the timer delays have to be scaled to allow the execution of the test, as shown in the following (fixed scaled timing):

$$
\begin{aligned}
S T & =S * N * \text { Tick, where } \\
S T & =\text { simulated timer delay } \\
S & =\text { scaling factor } \\
N * \text { Tick } & =\text { timer delay in the target } H W \text { environment }
\end{aligned}
$$


In practice, the scaling factor depends on the load $(L)$ and on the performance $(P)$ of the host. Therefore, (1) can be presented as follows:

$$
S T=S(L, P) * N * T i c k
$$

The equation (2) can be demonstrated quite easily, since the execution of a test case with a constant scaling factor $(S)$ for timer $(T)$, the test results produced by separate test runs are usually different. Sometimes, scaling requires changing during a test session, because different test cases are likely to have different timing requirements. At other times, a previously passed test case may not be passed, due to someone having started some heavy operation after logging onto the same host. Furthermore, new code in the tested software (such as new traces, instrumentation for measuring test coverage, or new features) may change the timing and cause the tests to fail due to the timers not having been adjusted to compensate for the added code. In this case, premature timeouts may occur.

It is very difficult to design a function capable of predicting the load and the performance in a reliable way. One possible solution is, instead of adjusting the scaling factor, letting it remain one, to scale the length of the simulated tick (STick) dynamically, while keeping the tick multiplier $(N)$ at the target environment value. Thus, (1) can be written as follows:

$$
\begin{aligned}
(1) S T & =S * N * \text { Tick; } \operatorname{Let}(S=1) \text { and }(\text { Tick }=\text { STick }) \\
\Rightarrow 1 * T & =N * \text { Stick } \\
\Rightarrow T & =N * \operatorname{Tick}(L, P)
\end{aligned}
$$

This allows the length of the tick to be scaled dynamically according to the load and the performance. This kind of timing is called dynamically scaled timing, involving dynamic adjustment of the length of the simulated tick during execution, in response to changes in the load and performance of the host (Figure 4). Since each tick is processed separately, this method can also be called continuous simulation. The fixed scaled timing involves scaling of the length of the target timer interrupt by a fixed multiplier, and therefore, the provided ticks are based on the clock of the host (i.e., the wall clock).

3.2.2 Discrete Simulation. The purpose of continuous simulation is to eliminate the effects of load and performance by scaling individual tick delays dynamically. However, in real systems, timer delays are usually longer than a single tick. This means that there is not necessarily anything happening in the test system during the individual ticks. Therefore, it can be estimated that improved performance may be achieved through a discrete handling of simulation time. This calls for an ability to relate the time advance to discrete steps (simulation cycles) instead of individual ticks. 


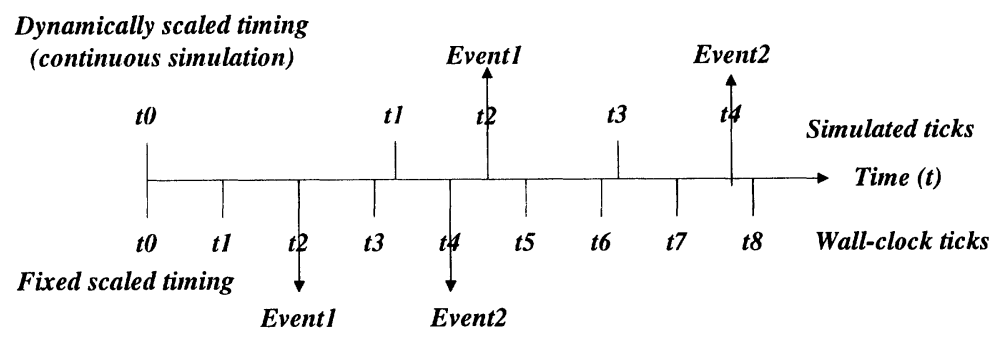

Figure 4 Continuous simulation and fixed scaled timing.

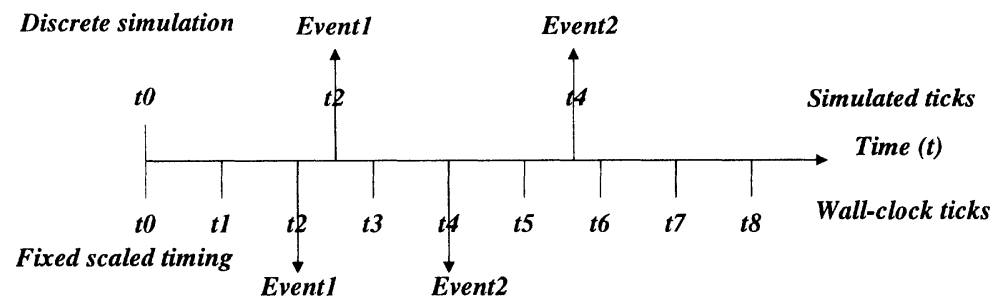

Figure 5 Discrete simulation and fixed scaled timing.

Discrete time simulation requires that each component of the test system has knowledge of the next expiring timer; this information can be used in defining the next step of simulation time. The tick multiplier $(N)$ in equation (3) can be utilised in defining the next simulation cycle. The discrete simulation time principle involves a continual adjustment of the length of each simulation cycle during execution, according to the next expiring timer of the test system. Therefore, simulation time advances in discrete steps first from $t 0$ to $t 2$, and then from $t 2$ to $t 4$, and so on (Figure 5).

\subsection{CONTROL OF SIMULATION TIME}

The control of simulation time is carried out by means of mode selection (Figure 6). The mode of simulation time can be local fixed scaled timing, global fixed scaled timing, sequential continuous timing, parallel continuous timing, deterministic continuous timing, or deterministic discrete timing. The features of the modes are described briefly in the following:

Local or global fixed scaled timing: Timing interrupts in each component in the test system are generated by one or more simulation clocks. Communication and timing signals between the test system components are allowed to form without restrictions, which means that external non-determinism is permitted. 
Sequential continuous timing: A global simulation clock generates timing interrupts in each component belonging to the test system in a predefined sequential order; ensuring that each component has processed the tick before it is sent to the next component. Thus, external non-determinism is reduced by means of a specific sequential order being predefined for the start of the event procedure(s), which are triggered by simulation clock ticks. However, some degree of external non-determinism is allowed, because the communication between test system components occurs without restrictions.

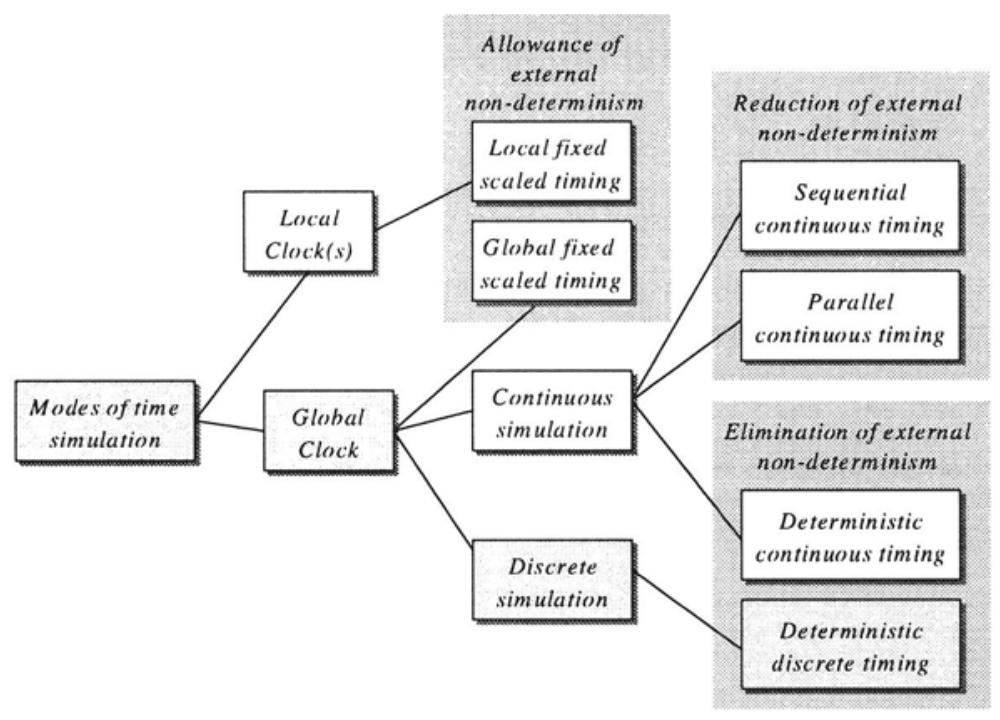

Figure 6 Control modes of simulation time.

Parallel continuous timing: Global simulation clock generates timing interrupts in parallel with all the components belonging to the test system. It also ensures that all the components have processed the tick before the next tick is sent. Thus, external non-determinism is reduced by forcing time to advance according to simulation clock ticks. However, timeouts may still occur in a non-deterministic order in the test system nodes, due to the fact that the ticks are delivered in parallel. In addition, communication between test system components is allowed to take place without restrictions.

Deterministic continuous timing: Global simulation clock works as a simulation scheduler, i.e., it delivers an execution token to each test system component at a given time and allows the component to accumulate its time by one tick. If any event procedures are triggered by the time increment, the system has to become stable before the time can advance. This allows elimi- 
nating external non-determinism and forcing concurrent behaviour to become sequential.

Deterministic discrete timing: Global simulation clock works as a simulation scheduler and delivers an execution token for each test system component at a time. It allows the component to accumulate its time by a specific amount of ticks $(\geq 1)$. If any event procedures are triggered by the time increment, the system has to become stable before the time can advance. This allows eliminating external non-determinism and forcing concurrent behaviour to become sequential.

In summary, in the elimination of external non-determinism, simulation scheduling serialises the communication between the test environment and the software under test. Then, only a partial order of events is tested. In reducing external non-determinism, simulation time is used to enhance the controllability of testing while allowing several possible orders for the events. By allowing external non-determinism, simulation time is used to scale timing in such a way that the tests can be executed in a host.

\subsection{ALGORITHM}

The basic idea of deterministic discrete timing is that time is not allowed to advance before all the events of the current simulation cycle have been completed and the system has reached a stable state.

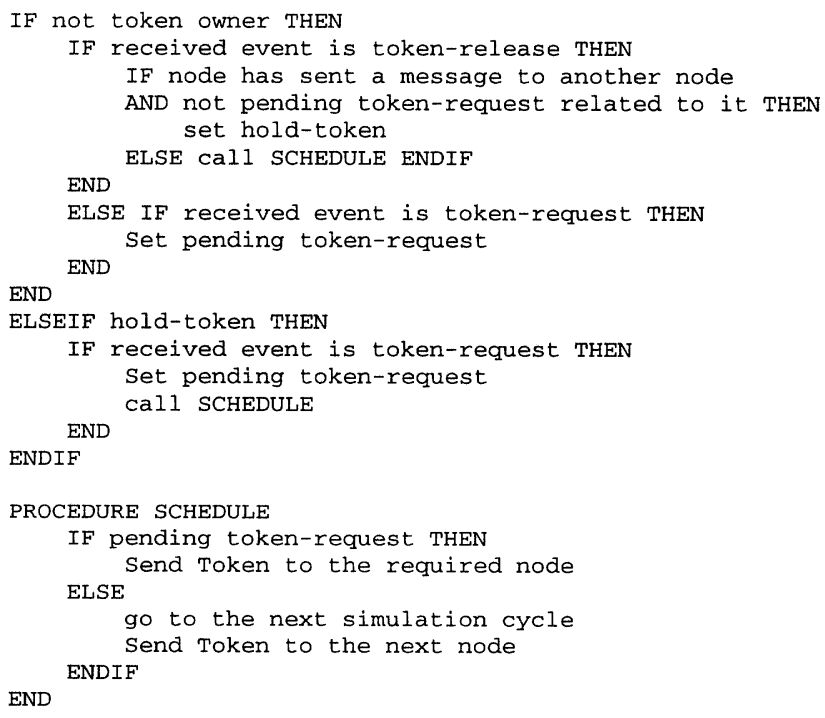

Figure 7 Deterministic discrete timing algorithm. 
A token is handed over to each component of the test system one at a time. In addition, a token may indicate timing advance, or just present an execution order to the component. The high-level control procedure of deterministic discrete timing is presented in Figure 7.

\section{CASE STUDY: TETRA TEST SYSTEM}

This chapter outlines a real-life system for testing the TETRA mobile terminal software on a HP-UX workstation, simulating the target system. Here, we are demonstrating how time simulation can be applied in conjunction with TTCN (ISO/IEC 9646-3) test cases and an SDL-based (ITU Z.100) implementation of the MCU software for the TETRA terminal.

The simulation of time and target system relies on MOSIM, which is a simulation-based tool and platform, designed for testing embedded software (Honka 1992, Latvakoski 1997). The time simulation concept and algorithms presented in this paper were implemented as parts of MOSIM libraries. The MOSIM libraries embody time simulation probes, offering a generic interface for the time simulation and scheduling algorithms. The following description focuses on depicting how the time simulation interfaces with TTCN and SDL.

\subsection{ARCHITECTURE}

The architecture of the TETRA test system is illustrated in Figure 8. The system comprises four main components and invisible write servers running as separate UNIX processes: TETRA Mobile MCU (software to be tested, IUT) and DSP Simulator, TETRA Base Station L2 Simulator, L3 TTCN Tester, and MOSIM Test Controller.

In Figure 8, the grey areas embrace the modules and functionality that enable the inter-working of the components. Communication takes place via the MOSIM protocol. The actual implementation of the protocol is based on asynchronous UNIX Domain or TCP/IP socket communication, as well as on the write server concept. The motivation of the write server concept is to guarantee deadlock-free communication and fair scheduling of the components. In addition to the reliable delivery of the application messages, MOSIM protocol takes care of the delivery of global timing, proper event ordering, well-controlled start-up and shut-down, and synchronous debugging support.

The behavioural part of ETS is produced by ITEX CCG (Telelogic 1998), which generates $\mathrm{C}$ code from TTCN ATS. The purpose of the TTCN Run-time System (RTS) is to interface ETS with the test system. RTS contains three interfaces: timer management, snapshot (PCO) queues, and sending/receiving events via PCOs. The $\mathrm{C}$ code generated by ITEX CCG provides hooks for the implementation of these interfaces. The majority of the L2 Simulator is generated by SDT CCG (Telelogic 1998) from the SDL system modelling 


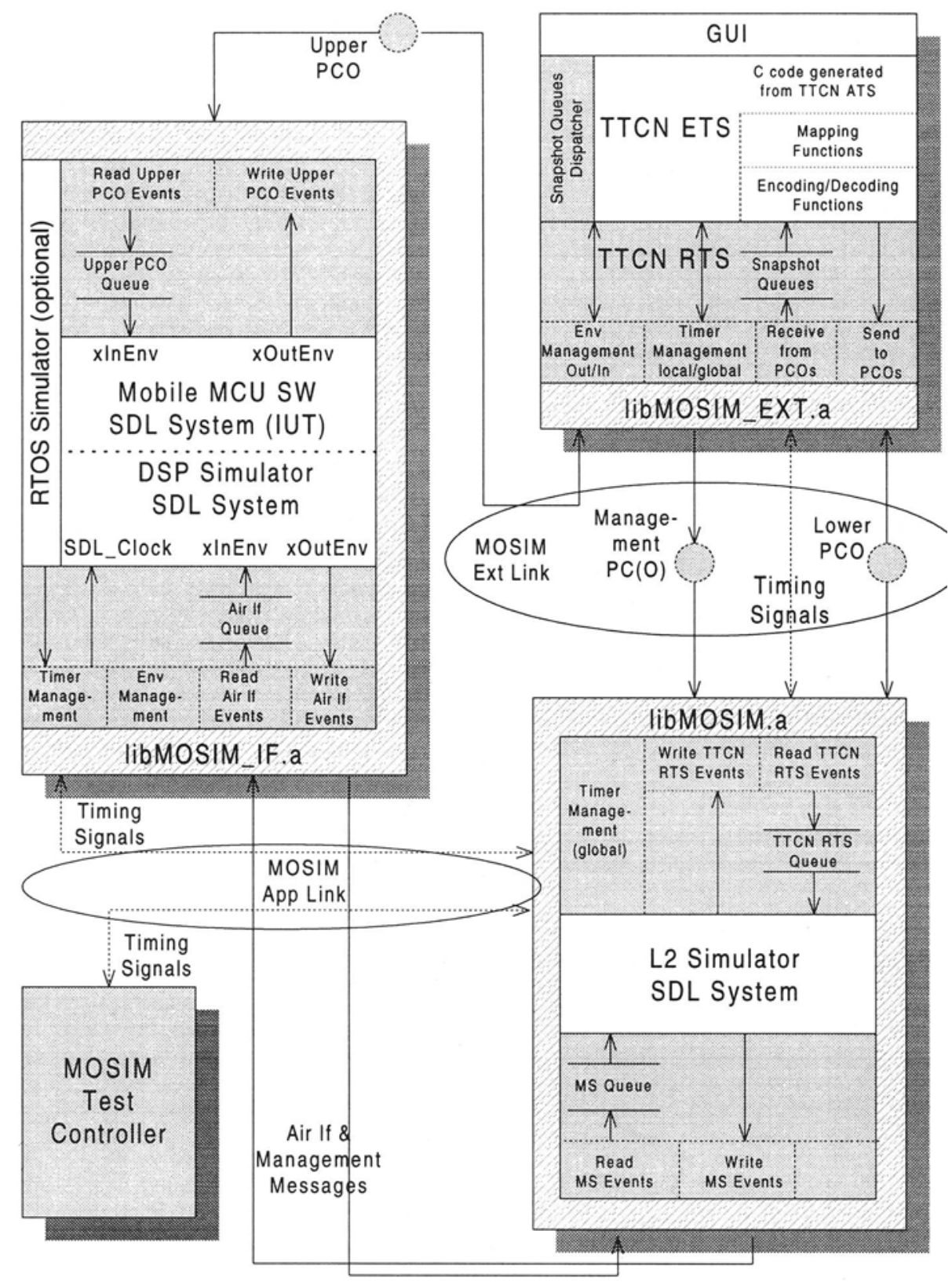

Figure 8 TETRA Test System Architecture. 
MAC, LLC and MM layers. SDT Simulator UI is optionally available for facilitating the debugging at the SDL level. The Mobile SDL System can be executed under the SDT Simulator, which implies that an SDT scheduler is applied. However, there is a disadvantage: the SDT scheduling policy is significantly different from that of RTOS, which implies that testing under SDT Simulator does not cover all the behaviour combinations that are possible on RTOS.

\subsection{TIMING MANAGEMENT}

Figure 9 depicts a timing scenario that may take place in the TETRA test system. This scenario and timing management are explained in the following, starting from the TTCN Tester interface and completing with the Mobile interface.

TTCN Tester Interface: TTCN RTS timer management is capable of working in both local and global timing modes. It contains two interface functions called by libMOSIM_EXT.a: one for switching between timing modes and the other for catching timing signals. In the local mode, the timer management is implemented as a delta list based on the use of the UNIX setitimer system service. In the global mode, the timing signals arrive from MOSIM. The arrival of a new timing signal is notified by invoking the interface function that instigates the appropriate actions with timer management, i.e., updating the timer delta list and launching the timer(s) expired. As soon as all actions belonging to the current time tick have been completed (i.e., TTCN RTS goes into an idle state), the timing signal is receipted by sending the idle signal to MOSIM. In practice, this means that the idle signal is sent as soon as the TTCN RTS goes on stand-by in the snapshot queue.

In the continuous timing algorithm, each timing signal has to be receipted separately. This may lead to a remarkable overhead when a massive amount of test cases are executed. This overhead can be decisively decreased by employing the discrete timing algorithm in which the moment of the next time-depended action (i.e., TTCN timer) is estimated and indicated.

L2 Simulator Interface: Actual timing signals are hidden from the L2 Simulator SDL System, because libMOSIM.a assumes control of handling those signals. The effect upon timing becomes implicitly true through the MOSIM simulation clock that is a part of libMOSIM.a. The current value of the simulated time is periodically inquired by the function SDL_Clock. The time passed to the SDL system is the simulated time, either local or global, depending on the MOSIM setting. The switching between different timing modes is internally managed by libMOSIM.a. Therefore, whether the timing mode is local or global, is not explicitly known by the L2 Simulator. 


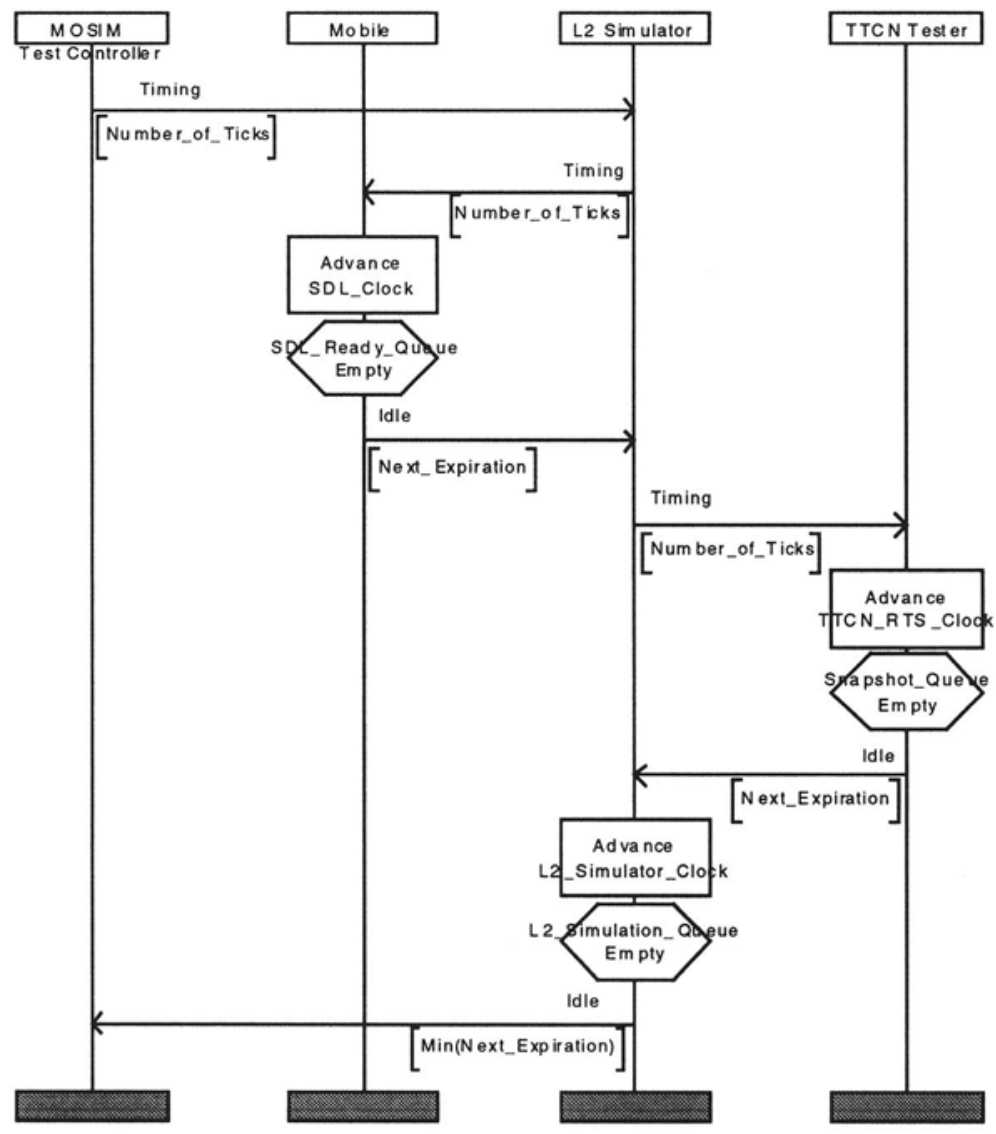

Figure 9 A timing scenario example.

Mobile SDL System Inteface: The timing modes available are, similarly to those of the TTCN Tester, local and global. In the local timing mode, the standard SDT implementation (i.e., high resolution time) of the function SDL_Clock is utilised (i.e., the SDL System is polling time by calling this function periodically from xMainLoop). In the global timing mode, the time value returned by SDL_Clock is based on the global clock provided by MOSIM. The switching between the timing modes is controlled by libMOSIM_IF.a and implemented in the functions of SDT_enable_timer_ticks (local time) and SDT_disable_timer_ticks (global time). According to the timing algorithm, an idle signal is sent to MOSIM by the SDL System as soon as all the activities scheduled for the current tick period have been completed. This is deduced from the status of the SDL Process ready queue by polling SDL_Transition_Prio in xInEnv. The idle signal is sent as soon as the ready queue is empty. The 
overhead arising from continuous timing signals can be decreased by employing the discrete timing algorithm in which the estimated moment of the next timedepended action (i.e., SDL timer) is returned to MOSIM as an idle signal parameter.

In the case of the RTOS Simulator, a standard MOSIM-RTOS timing interface is employed. In the local timing mode, the internal RTOS timer ticks are controlling the RTOS scheduler and timers. In the global timing mode, timer ticks are fed by MOSIM. Accordingly, an idle signal is sent by the RTOS Simulator as soon as all the activities scheduled for the tick period(s) have been completed.

\section{CONCLUDING REMARKS}

The time simulation methods described in this paper have been successfully employed in the integration testing of protocols for several standards, such as GSM and TETRA. The results indicate that the TTCN test sessions executed on a workstation simulating the target system are now repeatable and that tests can be executed automatically overnight. However, this requires a careful selection of the order of test execution. If an error is detected during a test session, the session will probably have to be aborted and restarted in order to prevent the emergence of potential side effects in the next test sets. The use of a global simulation clock has also facilitated the high-level controllability of debugging in distributed test systems by enabling the stopping of the time flow at exactly the increment of time, at which the software breakpoint occurs in the debugger (synchronous debugging). Furthermore, the demands arising from execution time no more restrict the capacity of observing time-critical lower protocol layers.

The developed system has turned out to be highly flexible, thanks to it allowing the selection of the time simulation mode according to the testing requirements. The main criteria for selection can be based on the behaviour of the target system, and, in particular, on whether the target system behaves in a non-deterministic way or not. However, the controllability of testing, the repeatability of tests, and the automating of test result analysis may require the use of a single global clock instead of a set of local clocks, so as to reduce non-determinism initially. The main benefit of the deterministic discrete timing algorithm can be found in the improved performance of the test system. For example, when using this algorithm, the execution time of an average test run of a GSM Phase 2 mobile terminal test system has been reduced to $68 \%$ of the time achieved with sequential continuous timing.

Acknowledgements: We would like to thank Mr. Juha Lehtikangas, Mr. Matti Sangi and Mr. Tero Manninen for their kind co-operation. 


\section{References}

Auer, A. \& Korhonen, J. 1995. State testing of embedded software. In: Conference Papers of 3rd European International Conference on Software Testing Analysis \& Review. EuroSTAR'95. Jacksonville, FL: Software Quality Engineering. $13 \mathrm{p}$.

Billoir, T. 1996. Methods and tools for developing distributed real-time systems. Real-Time Magazine. No. 2. Pp.73-78.

Bochmann, G. \& Bellal, O. B. 1990. Test result analysis with respect to formal specifications. In: de Meer, J., Mackert, L. \& Effelsberg, W (ed.) Protocol Test Systems II. The Netherlands: Elsevier Science Publishers B.V. Pp. 103117.

Bochmann, G., Desbiens, D., Dubuc, M., Ouimet, D. \& Saba, F. 1991. Test result analysis and validation of test verdicts. In: Davidson, I. \& Litwack, D. W. (ed.) Protocol Test Systems III. The Netherlands: Elsevier Science Publishers B.V. Pp. 263-274.

Candy, K. M. \& Misra, J. 1979. Distributed simulation: A case study in design and verification of distributed programs. IEEE Transactions on Software Engineering. Vol. 5, no. 5. Pp 440-452.

Carver, R. H. \& Tai, K. C. 1991. Replay and testing for concurrent programs. IEEE Software. Vol. 8, no. 2. Pp. 66-74.

Dotseth, M. \& Kuzara, E. 1993. Real-time debugging of embedded operating systems. In: Proceedings of the Sixth Annual Embedded Systems Conference. Volume 1. San Francisco, CA: Miller Freeman Inc. Pp. 217-226.

Fujimoto, R. M. 1995. Parallel and distributed simulation. In: Alexopoulos, C., Kang, K., Lilegdon, W. \& Goldsman, D. (ed.) Winter Simulation Conference Proceedings. San Diego, CA: WSC'95. Pp. 118-125.

ISO/IEC 9646 (1-5), 1991. Information technology - open systems interconnection - conformance testing methodology and framework. Ed 1. Geneva, Switzerland: IEC. 271 p. ITU Z.100 1994. Specification and description language (SDL). Ed 1. Geneva, Switzerland: ITU. 237 p.

Jefferson, D. R. 1985. Virtual time. ACM transactions on programming languages. Vol. 7, no. 3. 1985. Pp. 404-425.

Honka, H. 1992. A simulation-based approach to testing embedded software. Espoo: Technical Research Centre of Finland. 118 p. VTT Publications 124

Kim, M., Chanson, S.T. \& Yoo, S. 1995. Design for testability of protocols based on formal specifications. In: Cavalli, A. \& Budkowski, S. (ed.) Protocol Test Systems VIII. Proceedings of the IFIP WG6.1 TC6 8th International Workshop on Protocol Test Systems. Great Britain: Chapman \& Hall. Pp. 252-263.

Lamport, L. 1978. Time, clocks, and the ordering of events in a distributed system. Communications of the ACM. Vol. 21, no. 7. Pp 558-564. 
Latvakoski, J. 1997. Integration test automation of embedded communication software. Espoo: Technical Research Centre of Finland. 97 p. (VTT Publications 318 )

Liband, J. 1995. Techniques for real-time debugging. Embedded Systems Programming. Vol. 8, no. 4. Pp. 34-56.

de la Puente, J., Alonso, A., Leon, G. \& Duenas, J. C. 1993. Distributed execution of specifications. In: Real-Time Systems. Vol. 5, no 2-3. Pp. 213234.

de la Puente, J., Leon, G., Alonso, A., Viana, J. C., Ruz, M. A. \& Sink, E. W. 1994. Analysis of real-time object communication. IPTES Project EP5570 report IPTES-UPM-3-V2.6. Madrid, Spain: UPM. 57 p.

Sarikaya, B. 1988, Protocol test generation, trace analysis and verification techniques. In: Proceedings of the Second Workshop on Software Testing, Verification, and Analysis. Washington D.C.: IEEE Computer Society Press. Pp. 123-130.

Schütz, W. 1990. A test strategy for the distributed real-time system MARS. In: Proceedings of the COMPEURO ' 90 Conference. Washington D.C.: IEEE Computer Society Press. Pp. 20-27.

Seila, A. F. 1995. Introduction to simulation. In: Alexopoulos, C., Kang, K., Lilegdon, W. \& Goldsman, D. (ed.) Winter Simulation Conference Proceedings. San Diego, CA: WSC'95. Pp. 7-14.

Tai, K. C. 1985. On testing concurrent programs. Proceedings of COMPSAC '85. Chicago, IL: IEEE Computer Society Press. Pp. 310-317.

Tai, K. C., Carver, R. H. \& Obaid, E. E. 1991. Debugging concurrent Ada programs by deterministic execution. IEEE Transactions on Software Engineering. Vol. 17, no. 1. Pp. 45-63.

Taha, H. A., 1988. Simulation modeling and SIMNET. New Jersey: PrenticeHall Inc. 397p.

Telelogic 1998. Telelogic Tau 3.4 User's Manual.

Thome, B., 1993. Systems engineering: principles and practice of computerbased systems engineering. England: John Wiley \& Sons. 394 p.

Weiss, S. N. 1988. A formal framework for the study of concurrent program testing. In: Proceedings of the Second Workshop on Software Testing, Verification, and Analysis. Washington D.C.: IEEE Computer Society Press. Pp. 106-113.

Wvong, R. 1991. LAPB conformance testing using trace analysis. In: Jonsson, B., Parrow, J. \& Pehrson B. (ed.) Participants Proceedings of the 11th International IFIP WG6.1 Symposium on Protocol Specification, Testing, and Verification. Stockholm. Sweden. Pp. 248-261. 